Reprod. Nutr. Dévelop., 1985, 25 (1 B), 243-250.

\title{
Lipoprotéines plasmatiques du veau préruminant
}

\author{
D. BAUCHART, D. LEVIEUX $(*)$ \\ avec la collaboration technique de Marinette MEYER et Monique LEVIEUX
}

Laboratoire d'Etude du Métabolisme énergétique.

(*) Laboratoire des Maladies nutritionnelles,

I.N.R.A., Theix, 63122 Ceyrat, France.

Summary. Plasma lipoproteins of the preruminant calf.

The main lipoprotein classes (HDL, LDL, VLDL, chylomicrons) were isolated from the plasma of four 3-week old preruminant Friesian calves. The proportions of proteins and lipids and the fatty acid composition of the different lipid classes were determined over a 7-hour period corresponding to the pre and post-prandial periods. HDL, LDL, VLDL and chylomicrons averaged 58.3, 19.0, 13.3 and $9.1 \%$, respectively, of total lipoproteins. Triglycerides predominated in VLDL and chylomicrons, and cholesterol esters and phospholipids in HDL and LDL. The lipid proportion and fatty acid composition of a given lipoprotein were stable, except for the triglycerides of the chylomicrons (and VLDL) which were hydrolysed during a 2 to 5 -hour period after the morning meal, leading to a higher saturation of the remaining triglycerides. Linoleic acid, abundant in cholesterol esters of HDL $(46.5 \%)$ and LDL $(37.2 \%)$ and scarce in the cholesterol esters of VLDL $(3.5 \%)$ and chylomicrons $(3.2 \%)$, underlined the dual origin (plasma and tissues) of cholesterol esters in young bovines.

\section{Introduction.}

Les lipoprotéines de Bovin ont été largement étudiées depuis 15 ans chez l'animal adulte et principalement chez la Vache laitière car elles constituent à la fois la forme de transport des lipides utilisés pour le métabolisme énergétique de l'animal et une forme importante de transport des substrats nécessaires à la synthèse des matières grasses du lait par le tissu mammaire. La répartition et la structure chimique des lipides des différentes fractions lipoprotéiques ont été détaillées chez la Vache laitière haute productrice notamment par Stead et Welch $(1975,1976)$. Les variations de leurs caractéristiques ont été étudiées en fonction du cycle gestation-lactation (Raphael, Dimick et Puppione, 1973), de la traite (Gleockler, Ferreri et Flaim, 1980) ou chez la Vache cétonémique (Mc Carthy et al., 1968). Elles ont également été étudiées en fonction de différentes conditions nutritionnelles, notamment de la teneur de la ration en protéines (Stead et 
Oldham, 1978) en lipides non protégés (Dryden et al., 1971) ou en lipides protégés (Dryden et al., 1975).

Les lipoprotéines du jeune Veau ont, par contre, fait l'objet de peu d'études. Lascelles et Wadsworth (1971) ont caractérisé les lipoprotéines de la lymphe chez le Veau nouveau-né à jeun et plus récemment, Forte, Belle-Quint et Cheng (1981), en comparant les lipoprotéines du Veau fœtal et du Veau nouveau-né à celles de l'adulte, ont étudié les modifications de leur métabolisme au cours du développement.

Chez le Veau en croissance nourri exclusivement au lait maternel ou avec un aliment riche en matières grasses, Richard et al. (1980) puis Beynen et Van Gils (1983) ont, dans le cadre d'études expérimentales de l'athérosclérose humaine, étudié les effets de la supplémentation de la ration en cholestérol sur le taux de cholestérol des fractions lipoprotéiques mais n'ont effectué aucune mesure sur les autres fractions lipidiques des lipoprotéines chez cet animal.

Le but de ce travail est d'étudier chez le Veau préruminant âgé de 3 semaines recevant un aliment d'allaitement contenant $22 \%$ de matières grasses, la répartition et la composition des lipides des principales classes de lipoprotéines et de mesurer l'effet de la prise du repas sur l'évolution postprandiale de leur distribution plasmatique et des caractéristiques chimiques de leurs lipides.

\section{Matériel et méthodes.}

Animaux et aliment. - 4 veaux mâles de race frisonne ont reçu depuis l'âge de 1 jour et jusqu'à l'âge de 3 semaines et à raison de 2 repas égaux ( $8 \mathrm{~h}$ et $16 \mathrm{~h}$ ) par jour un aliment d'allaitement contenant par rapport à la matière sèche $22 \%$ de matières grasses sous forme de suif $(21 \%)$ et d'émulsifiant $(1 \%)$ et $65 \%$ de poudre de lait écrémée.

A l'âge de 3 semaines (âge correspondant aux prélèvements sanguins), le gain de poids vif des veaux était de $640 \pm 60 \mathrm{~g} / \mathrm{j}$ et la consommation journalière de matière sèche de $700 \pm 40 \mathrm{~g}$ pour un poids vif moyen de $45,7 \pm 3,5 \mathrm{~kg}$.

Prélèvements sanguins. - Les prélèvements sanguins ont été réalisés à l'âge de 3 semaines aux temps suivants : $2 \mathrm{~h}$ avant (T-2) puis $1 / 2(\mathrm{~T} 1 / 2), 2(\mathrm{~T} 2), 3(\mathrm{~T} 3)$, 5 (T5) et $7 \mathrm{~h}$ (T7) après la prise du repas du matin. Ils ont été effectués au niveau de la veine jugulaire à l'aide de tubes à prélèvement de $10 \mathrm{ml}$ sous vide et contenant de l'EDTA $(1 \mathrm{mg} / \mathrm{ml}$ de sang) et immédiatement centrifugés à 3000 $\mathrm{t} / \mathrm{m}$. Les plasmas récupérés $(10 \mathrm{ml})$ ont été conservés à $+4^{\circ} \mathrm{C}$ au maximum pendant $24 \mathrm{~h}$ avant d'être soumis au traitement de purification des lipoprotéines par ultracentrifugation. Une fraction aliquote $(10 \mathrm{ml})$ a été conservée à $-80^{\circ} \mathrm{C}$ pour les analyses de lipides du plasma total.

Préparation des fractions lipoprotéiques. - Les fractions lipoprotéiques ont été purifiées par ultracentrifugation préparative de flottaison selon une méthode dérivée de celle de Hatch et Lees (1968) avec une ultracentrifugeuse Beckman type L2 équipée du rotor $50 \mathrm{Ti}$. Les plasmas des veaux ont été traités individuellement et les fractions lipoprotéiques purifiées ont été récupérées quantitativement. 
La fraction I, correspondant aux chylomicrons (d $<1,000 \mathrm{~g} / \mathrm{ml}$ ) et aux VLDL $(1,000<d<1,006)$, est séparée de la fraction II, correspondant aux lipoprotéines de densité supérieure à 1,006, par centrifugation à $40000 \mathrm{tpm}$ pendant $18 \mathrm{~h}$ à $10^{\circ} \mathrm{C}$. Les chylomicrons sont purifiés à partir de la fraction I diluée dans du sérum physiologique à $15000 \mathrm{tpm}$ pendant $30 \mathrm{~min}$, puis les VLDL ont été purifiés à partir du sous-nageant ajusté à la densité de $1,006 \mathrm{~g} / \mathrm{ml}$ à l'aide de $\mathrm{KBr}$ solide, à 40000 tpm pendant $20 \mathrm{~h}$. Les $\operatorname{LDL}(1,006<\mathrm{d}<1,063)$ sont purifiées à partir de la fraction II, ajustée à d $=1,063$ avec $\mathrm{KBr}$, à 40000 tpm pendant $20 \mathrm{~h}$. Le sousnageant récupéré est ajusté à $d=1,210$ avec $\mathrm{KBr}$ et les $\mathrm{HDL}$ $(1,063<d<1,210)$ sont obtenues après centrifugation à $45000 \mathrm{tpm}$ pendant $25 \mathrm{~h}$.

La pureté des fractions lipoprotéiques a été contrôlée par électrophorèse sur gel de polyacrylamide en gradient discontinu de concentration et également sur plaque d'acétate de cellulose.

Analyses. - Les lipides du plasma et des fractions lipoprotéiques ont été extraits par le mélange chloroforme-méthanol selon la méthode de Folch, Lees et Sloane-Stanley (1957) après addition de 4 étalons internes (cholestéryl heptadécènoate, glycéryl mono et triheptadécènoate, acide heptadécènoique) synthétisés à partir de l'acide heptadécènoique purifié au laboratoire (Bauchart et Aurousseau, 1980). Les lipides ont été séparés par chromatographie sur couche mince de kieselgel délipidé dans le mélange hexane-éther éthylique-acide formique 80/20/2 (V/V/V). Après élution au chloroforme/méthanol $1 / 1(\mathrm{~V} / \mathrm{V})$, les lipides ont été saponifiés avec de la potasse alcoolique à $10 \%$ et leurs acides gras ont été méthylés puis analysés par chromatographie en phase gazeuse selon une méthode décrite par Bauchart et Aurousseau (1981). La teneur en cholestérol a été déterminée par voie enzymatique (méthode Chod-iodure, Meckotest). Les protéines ont été dosées par la méthode de Lowry en présence de Triton $X 100$ selon Kashyap, Hynd et Robinson (1980) afin d'éliminer le trouble de la solution colorée finale observé notamment avec les lipoprotéines de très basse densité.

\section{Résultats.}

La teneur et la répartition des principales lipoprotéines du Veau ainsi que leur composition en lipides et en protéines ont été mesurées en cinétique pré et postprandiale. Les moyennes de ces valeurs sont rapportées dans le tableau 1 . Les HDL constituent la classe prépondérante des lipoprotéines du Veau soit $58,3 \%$ des lipoprotéines totales $(64,2 \%$ chylomicrons déduits). La concentration en HDL baisse de $16 \%$ après le point T2 pour se maintenir ensuite (fig. 1). La fraction LDL ne représente que $19,0 \%$ des lipoprotéines totales $120,9 \%$ chylomicrons déduits) et leur teneur ne subit pas de variations significatives au cours de la journée (fig. 1). Par contre, les VLDL qui représentent $13,3 \%$ des lipoprotéines $(14,7 \%$ chylomicrons déduits $)$ subissent une baisse de $8 \% 2 \mathrm{~h}$ après la prise du repas puis réaugmentent ensuite avec un maximum $5 \mathrm{~h}$ après le repas $(+29,5 \%$ d'augmentation entre $\mathrm{T} 2$ et $\mathrm{T} 5)$. Les chylomicrons qui 


\section{TABLEAU 1}

Caractéristiques chimiques des principales classes de lipoprotéines plasmatiques chez le Veau préruminant âgé de 3 semaines (moyenne \pm écart-type à partir de 4 animaux et 6 points horaires) : teneur plasmatique des lipides et des protéines $(\mathrm{mg} / 100 \mathrm{ml}$ plasma) ; composition centésimale (valeurs entre parenthèses) des classes lipidiques individuelles (en \% du poids des lipides totaux) et des lipides et protéines totales (en \% du poids de la lipoprotéine).

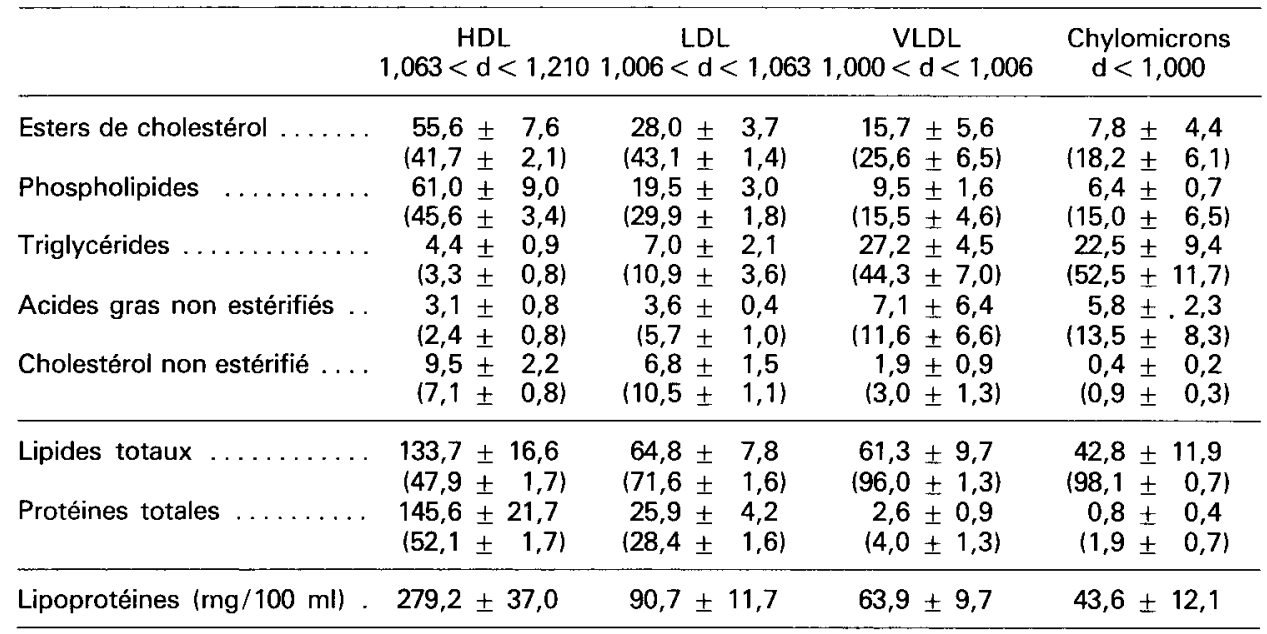

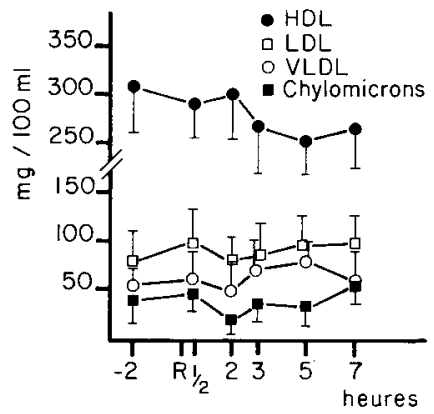

FIG. 1. - Variations pré- et post-prandiales des teneurs plasmatiques $(\mathrm{mg} / 100 \mathrm{ml})$ des principales classes de lipoprotéines chez le Veau préruminant âgé de 3 semaines.

représentent $9,1 \%$ des lipoprotéines totales suivent la même évolution pré et postprandiale mais de façon plus accentuée que les VLDL (fig. 1) : baisse de $51 \%$ entre les points T1/2 et T2 puis augmentation progressive avec un maximum $7 \mathrm{~h}$ après le repas $(+58,4 \%$ entre les points $\mathrm{T} 2$ et $\mathrm{T} 7, \mathrm{P}<0,01)$.

Les lipides des HDL $(44,7 \%$ des lipides totaux) sont riches en esters de cholestérol $(41,7 \%)$ et en phospholipides $(45,6 \%)$. La même tendance est observée avec les lipides des LDL $(21,4 \%$ des lipides totaux $)$ mais les phospholipides sont plus faiblement représentés $(29,9 \%)$. Dans le cas des VLDL, les lipides $(20,3 \%$ des lipides totaux) sont composés essentiellement de triglycérides $(44,3 \%)$ et secondairement d'esters de cholestérol $(25,6 \%)$. Enfin, les lipides des chylomicrons $(14,4 \%$ des lipides totaux) sont essentiellement représentés par les triglycérides $(52,5 \%)$ et secondairement par les esters de cholestéro! (18,2 \%) les phospholipides $(15,0 \%$ ) et les AGNE (13,5\%) (tabl. 1). La teneur en protéines des 
lipoprotéines est voisine de celle observée chez la plupart des mammifères : $52,1 \%$ pour les HDL, $28,4 \%$ pour les LDL, $4 \%$ pour les VLDL et $1,9 \%$ pour les chylomicrons (tabl. 1).

La composition en acides gras des phospholipides et des esters de cholestérol reste en moyenne relativement stable pour toutes les fractions lipoprotéiques au cours de la journée ; dans les HDL $(16,7 \%)$ et LDL $(16,8 \%)$, les phospholipides sont riches en C18:2 n- 6 mais ont un contenu en cet acide gras 3 fois plus faible dans les lipoprotéines de très basse densité ( $P<0,01$ ) (tabl. 2) ; la même tendance se vérifie avec les esters de cholestérol où les teneurs dans les HDL $(46,0 \%)$ et LDL $(37,2 \%)$ sont respectivement 13 et 10 fois plus élevées que dans les esters de cholestérol des lipoprotéines plus légères (tabl. 3).

Dans le cas des triglycérides, majoritaires dans les VLDL $(44,5 \%)$ et les chylomicrons $(36,8 \%)$, la composition en acides gras varie très étroitement avec leur teneur plasmatique (tabl. 4 ; fig. 2). De composition très voisine de celle des

\section{TABLEAU 2}

Composition en acides gras (en \% en poids des esters méthyliques d'acides gras) des phospholipides des principales classes de lipoprotéines du Veau préruminant (moyenne \pm écart type ; 4 animaux et 6 points horaires).

\begin{tabular}{ccrrr}
\hline & HDL & LDL & VLDL & Chylomicrons \\
\hline C16:0 & $12,8 \pm 1,6$ & $19,3 \pm 2,1$ & $30,4 \pm 2,0$ & $33,4 \pm 3,4$ \\
C18:0 & $29,6 \pm 3,4$ & $22,8 \pm 3,3$ & $23,1 \pm 1,9$ & $24,6 \pm 1,7$ \\
C18:1 & $27,3 \pm 2,0$ & $23,3 \pm 2,7$ & $18,3 \pm 1,3$ & $16,0 \pm 2,9$ \\
C18:2 & $16,7 \pm 2,4$ & $16,8 \pm 3,0$ & $5,3 \pm 1,0$ & $5,1 \pm 1,1$ \\
\hline
\end{tabular}

TABLEAU 3

Composition en acides gras (en \% en poids des esters méthyliques d'acides gras) des esters de cholestérol des principales classes de lipoprotéines du Veau préruminant (moyenne \pm écart type ; 4 animaux et 6 points horaires).

\begin{tabular}{rrrrr} 
& HDL & \multicolumn{1}{c}{ LDL } & \multicolumn{1}{c}{ VLDL } & Chylomicrons \\
\hline C16:0 & $11,2 \pm 1,7$ & $16,0 \pm 1,7$ & $29,0 \pm 1,7$ & $26,5 \pm 3,8$ \\
C18:0 & $1,9 \pm 0,4$ & $5,4 \pm 0,7$ & $24,3 \pm 6,2$ & $22,3 \pm 3,7$ \\
C18:1 & $13,8 \pm 2,1$ & $19,9 \pm 1,3$ & $11,1 \pm 2,3$ & $14,5 \pm 4,3$ \\
C18:2 & $46,0 \pm 5,6$ & $37,2 \pm 5,8$ & $3,5 \pm 0,8$ & $3,2 \pm 1,1$
\end{tabular}

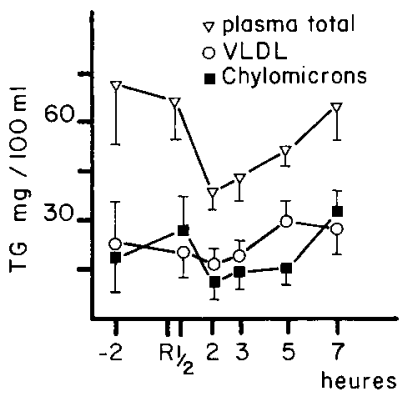

FIG. 2. - Variations pré- et post-prandiales des teneurs plasmatiques $(\mathrm{mg} / 100 \mathrm{ml})$ des triglycérides du plasma total, des VLDL et des chylomicrons chez le Veau préruminant âgé de 3 semaines.

Reproduction Nutrition Développement, $n^{\circ} 1$ B-1985. - 7 
triglycérides alimentaires aux points $T-2$ et $T+1 / 2$, les triglycérides des VLDL et surtout des chylomicrons subissent pendant la période T2-T3 une forte dégradation accompagnée d'une forte saturation des triglycérides restants. Cette tendance s'inverse complètement $(P<0,05) 7 \mathrm{~h}$ après le repas avec notamment une élévation des teneurs en triglycérides des chylomicrons $(+73,3 \%$ entre T2 et T7) et la reprise d'une composition en acides gras identique à celle des triglycérides alimentaires (tabl. 4).

\section{TABLEAU 4}

Effet de la prise du repas sur la composition en acides gras

(en $\%$ en poids des esters méthyliques d'acides gras) des triglycérides des chylomicrons et des $V L D L$ du Veau préruminant (moyenne \pm écart type ; 4 animaux).

Comparaison avec les triglycérides du plasma total et de l'aliment.

\begin{tabular}{|c|c|c|c|c|c|c|c|c|}
\hline \multirow{2}{*}{\multicolumn{2}{|c|}{$\begin{array}{c}\text { Acides } \\
\text { gras }\end{array}$}} & \multicolumn{6}{|c|}{ Heure par rapport au repas } & \multirow[b]{2}{*}{ Aliment } \\
\hline & & $-2 h$ & $+1 / 2 h$ & $+2 h$ & $+3 h$ & $+5 h$ & $+7 \mathrm{~h}$ & \\
\hline C16:0 & $\left\{\begin{array}{l}\text { Chylomicrons } \\
\text { VLDL } \\
\text { Plasma total }\end{array}\right.$ & $\begin{array}{l}20,1 \pm 1,2 \\
30,5 \pm 1,7 \\
24,2 \pm 1,8\end{array}$ & $\begin{array}{l}23,9 \pm 0,9 \\
24,6 \pm 1,5 \\
25,6 \pm 1,3\end{array}$ & $\begin{array}{l}29,6 \pm 2,1 \\
28,5 \pm 1,7 \\
25,2 \pm 1,6\end{array}$ & $\begin{array}{l}24,8 \pm 1,5 \\
30,5 \pm 1,6 \\
25,9 \pm 1,0\end{array}$ & $\begin{array}{l}28, \pm 1,2 \\
32,9 \pm 2,5 \\
32,2 \pm 1,0\end{array}$ & $\begin{array}{l}27,8 \pm 1,8 \\
24,9 \pm 2,0 \\
25,9 \pm 1,8\end{array}$ & 23,9 \\
\hline C18:0 & $\left\{\begin{array}{l}\text { Chylomicrons } \\
\text { VLDL } \\
\text { Plasma total }\end{array}\right.$ & $\begin{array}{l}12,3 \pm 1,5 \\
20,3 \pm 1,6 \\
16,0 \pm 1,5\end{array}$ & $\begin{array}{l}18,3 \pm 0,9 \\
19,2 \pm 1,2 \\
17,9 \pm 1,3\end{array}$ & $\begin{array}{l}21,4 \pm 1,2 \\
19,3 \pm 1,5 \\
16,5 \pm 1,7\end{array}$ & $\begin{array}{l}18,2 \pm 1,0 \\
20,0 \pm 1,7 \\
18,2 \pm 1,5\end{array}$ & $\begin{array}{l}20,9 \pm 1,1 \\
21,0 \pm 2,3 \\
22,6 \pm 1,9 \\
\end{array}$ & $\begin{array}{l}19,6 \pm 1,7 \\
17,4 \pm 1,5 \\
18,4 \pm 2,0\end{array}$ & 20,5 \\
\hline C18:1 & $\left\{\begin{array}{l}\text { Chylomicrons } \\
\text { VLDL } \\
\text { Plasma total }\end{array}\right.$ & $\begin{array}{l}43,7 \pm 2,1 \\
29,2 \pm 1,9 \\
38,5 \pm 2,0\end{array}$ & $\begin{array}{l}38,8 \pm 1,5 \\
33,5 \pm 1,7 \\
36,1 \pm 1,9\end{array}$ & $\begin{array}{l}26,4 \pm 1,8 \\
21,7 \pm 2,0 \\
27,9 \pm 1,7\end{array}$ & $\begin{array}{l}24,2 \pm 2,0 \\
17,7 \pm 1,9 \\
25,2 \pm 1,4\end{array}$ & $\begin{array}{l}16,8 \pm 1,6 \\
10,1 \pm 1,7 \\
12,3 \pm 0,9\end{array}$ & $\begin{array}{l}36,4 \pm 2,0 \\
29,0 \pm 2,1 \\
35,7 \pm 1,8\end{array}$ & 38,6 \\
\hline
\end{tabular}

\section{Discussion. Conclusion.}

La répartition plasmatique des lipoprotéines du Veau préruminant se caractérise, comme chez le Bovin adulte (Stead et Welch, 1975 ; Forte, Bell-Quint et Cheng, 1981) par l'abondance des HDL $(58,3 \%)$ et leur relative pauvreté en LDL $(19,0 \%)$. Cependant, par l'ingestion de lait riche en matières grasses $(22 \%)$, le Veau accumule, à la différence de l'adulte $(<5 \%), 22,4 \%$ de lipoprotéines de très basse densité dont $9,2 \%$ sous forme de chylomicrons qui représentent la forme essentielle du transport des lipides alimentaires utilisables par les tissus. Comme l'Agneau préruminant (Leat, Kubassek et Butress, 1976), le Bovin peut synthétiser des chylomicrons à l'état préruminant et conserver vraisemblablement la possibilité d'assurer la même synthèse à l'état adulte si la quantité et la nature insaturée des lipides absorbés est suffisante. En effet, Harrison, Leat et Forster (1974) ont montré que le Mouton adulte synthétise de fortes quantités de chylomicrons si l'on infuse directement dans le duodénum des triglycérides fortement insaturés (huile de maïs).

Le contenu en lipides et en protéines des lipoprotéines du Veau est voisin de celui du Bovin adulte (Stead et Welch, 1975 ; Forte, Bell-Quint et Cheng, 1981) et de I'Homme (Fruchart et Sezille, 1977) sauf dans le cas des VLDL où le Veau ne 
possède que $4 \%$ de protéines $(6 \%$ chez l'adulte, Stead et Welch, 1975) contre $10 \%$ environ chez l'Homme. De même, le plus faible contenu en triglycérides des chylomicrons $(52,5 \%)$ par rapport à ceux de l'Homme $178 \%$, Fruchart et Sezille, 1977) semble être une caractéristique des préruminants puisque Leat, Kubasek et Butress (1976) avaient enregistré la même tendance avec les lipoprotéines très légères de l'Agneau nourri au lait.

La grande richesse en C18:2 n-6 des esters de cholestérol des HDL et LDL confirme la haute spécificité de la lécithine-cholestérol Acyl transférase (LCAT) bovine pour les acides gras polyinsaturés liés en position $\beta$ des lécithines, mise en évidence in vitro par Noble, O'Kelly et Moore (1972). D'après ces auteurs, la LCAT bovine se distinguerait de la LCAT humaine (Glomset et Norum, 1973) ou de Rat (Sugano et Portman, 1964) par la non-possibilité de transférer des acides gras saturés ou monoinsaturés. Les molécules de cholestérol estérifiées avec du palmitate ou de l'oléate dans les HDL et LDL mais surtout dans les lipoprotéines très légères, seraient dues à l'action des Acyl-cholesterol Acyl transférase (ACAT) tissulaires et notamment hépatique mises en évidence chez le Veau par Stokke (1974). D'après Noble, Crouchman et Moore (1975) l'ACAT ovine apparaît non spécifique vis-à-vis de la nature de chaîne des Acyl CoA, ce qui expliquerait chez le Veau préruminant comme chez l'adulte (Dryden et al., 1971 ; Stead et Welch, 1975) la très faible teneur en acides gras polyinsaturés des esters de cholestérol des lipoprotéines très légères. Cette tendance pourrait être accrue dans notre expérience par la faible teneur en C18:2 n-6 de la ration.

L'évolution postprandiale des teneurs et de la composition des triglycérides a été décrite précédemment (Bauchart et Aurousseau, 1981). La présente expérience montre que cette évolution dépend de façon quasi-exclusive des triglycérides des lipoprotéines très légères. La baisse de la triglycéridémie $2 \mathrm{~h}$ après le repas est due principalement à la dégradation des triglycérides des chylomicrons en accord avec la spécificité plus grande de la LPL par ceux-ci (Fieldding et Higgins, 1974) et secondairement à ceux des VLDL. Au cours de cette période, le sang s'enrichit en résidus lipoprotéiques dont les triglycérides seraient plus saturés ce qui soulignerait l'affinité plus grande des LPL du bovin comme celles de I'Homme (Skipski, 1972) pour les lipoprotéines très légères de fort diamètre riches en triglycérides insaturés. Par contre, l'apparition des VLDL riches en acides gras saturés $5 \mathrm{~h}$ après le repas pose le problème d'une émission plus importante de VLDL par le foie ce qui est actuellement en cours de vérification.

$10^{e}$ Réunion du groupe Développement I.N.R.A., Rennes, 9-10 mai 1984.

\section{Références}

BAUCHART D., AUROUSSEAU B., 1980. Preparation of heptadecenoic acid from Candida tropicalis yeast. J. am. Oil Chem. Soc., 57, 121-124.

BAUCHART D., AUROUSSEAU B., 1981. Posptrandial lipids in blood plasma of preruminant calves. J. Dairy Sci., 64, 2033-2042.

BEYNEN A. C., Van GILS L. G. M., 1983. Composition of serum lipoproteins in veal calves fed a milk replacer supplemented with cholesterol. Nutr. Report Int., 27, 587-597. 
DRYDEN F. D., MARCHELLO J. A., ADAMS G. H., HALE W. H., 1971. Bovine serum lipids. II. Lipoprotein quantitative and qualitative composition as influenced by added animal fat diets. J. anim. Sci., 32, 1016-1029.

DRYDEN F. D., MARCHELLO J. A., CUITUN L. L., HALE W. H., 1975. Protein protected fat for ruminants. II. Serum lipids and lipoproteins. J. anim. Sci., 40, 697-705.

FIELDING C. J., HIGGINS J. M., 1974. Lipoprotein lipase: comparative properties of the membrane-supported and solubilized enzyme species. Biochemistry, 13, 4324-4330.

FOLCH J., LEES M., SLOANE-STANLEY G. H., 1975. A simple method for the isolation and purification of total lipids from animal tissues. J. biol. Chem., 226, 497-509.

FORTE T. M., BELL-QUINT J., CHENG F., 1981. Lipoproteins of fetal and new-born calves and adult steer : a study of developmental changes. Lipids, 16, 240-245.

FRUCHART J. C., SEZILLE G., 1977. Transport et destinée des lipides plasmatiques chez I'Homme normal. Rev. franc. Corps gras, 24, 151-159.

GLEOCKLER D. H., FERRERI L. F., FLAIM E., 1980. Lipoprotein in normal lactating cows bled at various times : effect of milking. Proc. Soc. exp. Biol. Med., 165, 118-122.

GLOMSET J. A., NORUM K. R., 1973. The metabolic role of lecithin : cholesterol acyl transferase. Perspectives from pathology, 1-65. In R. PAOLETTTI, D. KRITCHEVSKY, Advances in Lipid Research, 11, Acad. Press., N.Y.

HARRISON F. A., LEAT W. M. F., FORSTER A., 1974. Absorption of maize oil infused into the duodenum of the sheep. Proc. Nutr. Soc., 33, 101 A.

HATCH F. T., LEES R. S., 1968. Pratical methods for plasma lipoprotein analysis. Advan. Lipid Res., 6, 1-68.

KASHYAP M. L., HYND B. A., ROBINSON K., 1980. A rapid and simpie method for measurement of total protein in very low density lipoproteins by the lowry assay. J. Lipid Res., 21, 491-495.

LASCELLES A. K., WADSWORTH J. C., 1971. The origin of lipoprotein in the intestinal and hepatic lymph of unsuckled new-born calves. J. Physiol., 214, 443-455.

LEAT W. M. F., KUBASEK F. O. T., BUTRESS N., 1976. Plasma lipoproteins of lambs and sheep. Quart. J. exp. Physiol., 61, 193-202.

NOBLE R. C., O'KELLY J. C., MOORE J. C., 1972. Observations on the lecithin : cholesterol acyl transferase system in bovine plasma. Bioch. biophys. Acta, 270, 519-528.

NOBLE R. C., CROUCHMAN M. L., MOORE J. H., 1975. Synthesis of cholesterol ester in the plasma and liver of sheep. Lipids, 10, 790-799.

McCARTHY R. D., CHANDLER P. T., GRIEL L. C. Jr., PORTER G. A., 1968. Fatty acid composition of blood serum lipoproteins from normal and ketonic cows. J. Dairy Sci., 51, 392-396.

RAPHAEL B. C., DIMICK P. S., PUPPIONE D. L., 1973. Lipid characterization of bovine serum lipoproteins throughout gestation and lactation. J. Dairy Sci., 56, 1025-1032.

RICHARD M. J., STEWART J. W., HEEG T. R., WIGGERS K. D., JACOBSON N. L., 1980. Blood plasma lipoprotein and tissue cholesterol of calves fed soy-bean oil, corn oil, vegetable shortening or tallow. Atherosclerosis, 37, 513-520.

SKIPSKI V. P., 1972. Lipid composition of lipoproteins in normal and diseased states, 471-583. In NELSON G. Blood lipids and lipoproteins : quantification, composition and metabolism. Wiley Interscience, N.Y. et London.

STEAD D., WELCH V. A., 1975. Lipid composition of bovine serum lipoproteins. J. Dairy Sci., 58, $122-127$.

STEAD D., WELCH V. A., 1976. Determination of physical properties of bovine serum lipoproteins by analytical ultracentrifugation. J. Dairy Sci., 59, 9-13.

STEAD D., OLDHAM J. D., 1978. The effects of low-and high-protein diets upon the concentrations of plasma low density lipoproteins in Friesian heifers throughout lactation. Proc. Nutr. Soc., 37, 45A.

STOKKE K. T., 1974. Cholesteryl ester metabolism in liver and blood plasma of various animal species. Atherosclerosis, 19, 393-406.

SUGANO M., PORTMAN O. W., 1964. Fatty acid specificities and rates of cholesterol esterification in vivo and in vitro. Arch. Biochem. Biophys., 107, 341-350. 